\title{
Relating molecular structure and low-energy fusion through time-dependent wave-packet dynamics: the ${ }^{12} \mathrm{C}+{ }^{12} \mathrm{C}$ collision
}

\author{
Alexis Diaz-Torres ${ }^{1, a}$ and Michael Wiescher ${ }^{2, b}$ \\ ${ }^{1} E_{C} T^{*}$, Villa Tambosi, 38123 Villazzano, Trento, Italy \\ ${ }^{2}$ JINA and University of Notre Dame, Indiana 46656, USA
}

\begin{abstract}
Recent progress in a quantitative study of the ${ }^{12} \mathrm{C}+{ }^{12} \mathrm{C}$ sub-Coulomb fusion is reported. It is carried out using full-dimensional, time-dependent wave-packet dynamics, a quantum reaction model that has not been much exploited in nuclear physics, unlike in chemical physics. The low-energy collision is described in the rotating center-of-mass frame within a nuclear molecular picture. A collective Hamiltonian drives the time propagation of the wave-packet through the collective potential-energy landscape that is calculated with a realistic two-center shell model. Among other preliminary results, the theoretical sub-Coulomb fusion resonances for ${ }^{12} \mathrm{C}+{ }^{12} \mathrm{C}$ seem to correspond well with observations. The method appears to be useful for expanding the cross-section predictions towards stellar energies.
\end{abstract}

\section{Introduction}

The physics of low-energy nuclear reactions is crucial for understanding the chemical evolution of the Universe [1]. For instance, the ${ }^{12} \mathrm{C}+{ }^{12} \mathrm{C}$ fusion at energies near the Gamow peak $(\sim 1.5 \mathrm{MeV})$ plays a key role in the stellar carbon burning, whose cross section is commonly determined by extrapolating high-energy fusion data [2-4]. The direct fusion measurements are very difficult to carry out at very low energies $(\leq 3 \mathrm{MeV})$, the observed resonant structures making the extrapolation very uncertain [5-7].

The sub-Coulomb fusion of ${ }^{12} \mathrm{C}+{ }^{12} \mathrm{C}$ has been recently addressed with the coupled channels method [8,9]. These calculations suggest important effects of both the low-lying low-density energy spectrum of ${ }^{24} \mathrm{Mg}$ and the ${ }^{12} \mathrm{C}$ Hoyle state on the low-energy fusion cross section. The theoretical fusion excitation curves are smooth, without resonant structures. This feature seems to be due to the use of a strong absorption model that does not include the physics of intermediate structure (nuclear molecule) [10]. The key role of intermediate structure in fusion can be addressed with a new quantitative model [11]. Recent progress in this new model is reported in the present contribution.

\section{New fusion model}

Figure 1 shows the ${ }^{12} \mathrm{C}+{ }^{12} \mathrm{C}$ nuclear molecule (upper panel) along with the collective potential-energy landscape (lower panel) as a function of the internuclear distance and the alignment between the two deformed nuclei. The potential curves are presented for fixed orientation of the

\footnotetext{
a e-mail: torres@ectstar.eu

be-mail: michael.c.wiescher.1@nd.edu
}

two ${ }^{12} \mathrm{C}$ intrinsic symmetry axis relative to the internuclear axis; the three axes are coplanar. The potential of the non-axial symmetric configurations very weakly depends on the azimuthal angle of the ${ }^{12} \mathrm{C}$ intrinsic symmetry axis. The collective potential energy has been calculated with the finite-range liquid-drop model [12] using nuclear shapes of a realistic two-center shell model [13]. The large oblate deformation of ${ }^{12} \mathrm{C}\left(\beta_{2}=-0.5\right.$ and moment of inertia $I=0.67 \hbar^{2} \mathrm{MeV}^{-1}$ which is consistent with the observed $2^{+}$excitation energy $\left[E_{2^{+}}=\frac{\hbar^{2}}{2 I} 2(2+1)=4.43\right.$ $\mathrm{MeV}])$ results in a continuous of Coulomb barriers and potential pockets, which are distributed in a range of radii ( $R=4-8 \mathrm{fm})$. The lowest barrier $(90-90$ alignment $)$ favors the initial approach of ${ }^{12} \mathrm{C}$ nuclei which must reorientate in order to get trapped in the deepest pocket of the potential $(0-0$ alignment $)$ where fusion occurs. In transit to fusion, the ${ }^{12} \mathrm{C}+{ }^{12} \mathrm{C}$ nuclear molecule can populate quasi-stationary (doorway) states belonging to the shallow potential pockets of the non-axial symmetric configurations. These doorway states may also decay into scattering states, instead of feeding fusion, as the ${ }^{12} \mathrm{C}$ nuclei largely keep their individuality within the molecule [13]. The complex motion of the ${ }^{12} \mathrm{C}+{ }^{12} \mathrm{C}$ nuclear molecule through the potential energy landscape is driven by the kinetic energy operator that includes Coriolis interaction between the total angular momentum of the system and the intrinsic angular momentum of the ${ }^{12} \mathrm{C}$ nuclei. We use an exact expression of the kinetic energy operator [14].

Having determined the total collective Hamiltonian of the ${ }^{12} \mathrm{C}+{ }^{12} \mathrm{C}$ system in terms of the radial coordinate, $R$, and the spherical angles of the ${ }^{12} \mathrm{C}$ symmetry axis, $\theta_{i}$ and $\phi_{i}$, the time propagation of an initial wave function has been carried out using the modified Chebyshev propagator 

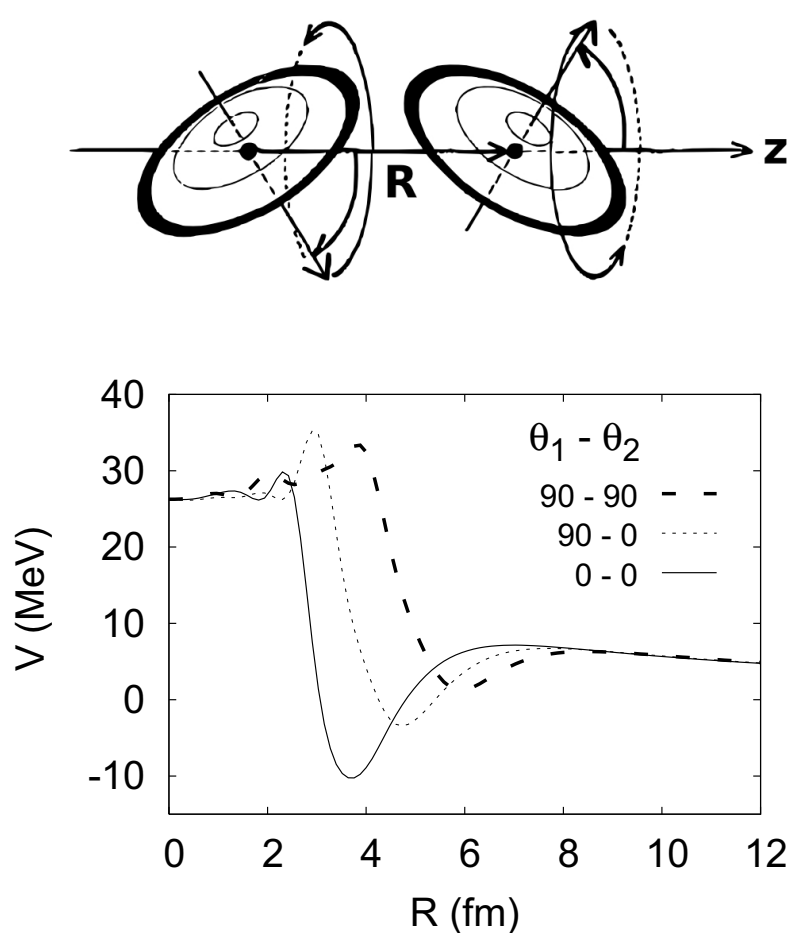

Figure 1. Cuts in the collective potential-energy landscape for the ${ }^{12} \mathrm{C}+{ }^{12} \mathrm{C}$ nuclear molecule as a function of the internuclear distance and alignment, $V\left(R, \theta_{1}, \phi_{1}=0, \theta_{2}, \phi_{2}=0\right)$. The 90 90 alignment (dashed line) facilitates the access by tunneling to the potential pockets. These are explored by the system, guided by the kinetic-energy operator which drives non-axial symmetric configurations towards the potential pocket of 0-0 alignment (solid line), where fusion happens by a strong absorption.

for the evolution operator [15]. The initial wave function is determined when the ${ }^{12} \mathrm{C}$ nuclei are far apart at their ground-states $\left(0^{+}\right)$, the radial and the internal coordinates being decoupled:

$$
\Psi_{0}\left(R, \theta_{1}, k_{1}, \theta_{2}, k_{2}\right)=\chi_{0}(R) \psi_{0}\left(\theta_{1}, k_{1}, \theta_{2}, k_{2}\right),
$$

where $k_{i}$ are conjugate momenta of the $\phi_{i}$ azimuthal angles, so (1) is in a mixed representation. The radial component, $\chi_{0}(R)$, is considered a Gaussian wave-packet, while $\psi_{0}\left(\theta_{1}, k_{1}, \theta_{2}, k_{2}\right)$ is the internal symmetrized wave function due to the exchange symmetry of the system:

$$
\begin{array}{r}
\psi_{0}\left(\theta_{1}, k_{1}, \theta_{2}, k_{2}\right)=\left[\zeta_{j_{1}, m_{1}}\left(\theta_{1}, k_{1}\right) \zeta_{j_{2}, m_{2}}\left(\theta_{2}, k_{2}\right)\right. \\
\left.+(-1)^{J} \zeta_{j_{2},-m_{2}}\left(\theta_{1}, k_{1}\right) \zeta_{j_{1},-m_{1}}\left(\theta_{2}, k_{2}\right)\right] \\
/ \sqrt{2+2 \delta_{j_{1}, j_{2}} \delta_{m_{1},-m_{2}}},
\end{array}
$$

where $\zeta_{j, m}(\theta, k)=\sqrt{\frac{(2 j+1)(j-m) !}{2(j+m) !}} P_{j}^{m}(\cos \theta) \delta_{k m}$, and $P_{j}^{m}$ are associated Legendre functions. At the ${ }^{12} \mathrm{C}$ ground-state, $j_{1}=j_{2}=0$ and $m_{1}=m_{2}=0$. Please note that the radial and internal coordinates are strongly coupled when the ${ }^{12} \mathrm{C}$ nuclei come together, so a product state like Eq. (1) is only justified asymptotically.

Since the initial radial wave-packet, $\chi_{0}(R)$, contains different translational energies, an energy projection

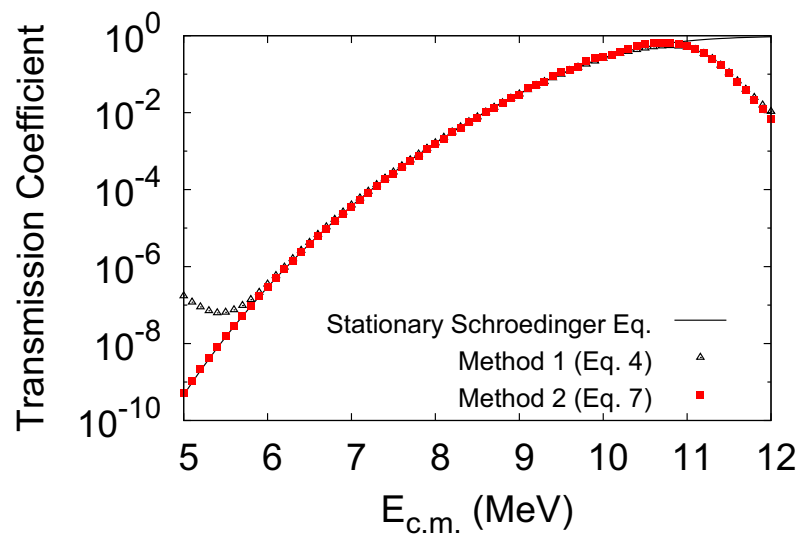

Figure 2. Transmission-coefficient excitation function for the ${ }^{16} \mathrm{O}+{ }^{16} \mathrm{O}$ central collision through the Coulomb barrier of the Broglia-Winther potential, calculated with various methods indicated. The Coulomb-barrier energy is about $10 \mathrm{MeV}$.

method is required. The energy-resolved scattering information can be obtained using a window operator [16]. The key idea is to calculate the energy spectrum, $\mathcal{P}\left(E_{k}\right)$, of the initial and final wave functions. $E_{k}$ is the centroid of a total energy bin of width $2 \epsilon$. A vector of reflection coefficients, $\mathcal{R}\left(E_{k}\right)$, is determined by the ratio [17]:

$$
\mathcal{R}\left(E_{k}\right)=\frac{\mathcal{P}^{\text {final }}\left(E_{k}\right)}{\mathcal{P}^{\text {initial }}\left(E_{k}\right)} .
$$

The transmission coefficients are:

$$
\mathcal{T}\left(E_{k}\right)=1-\mathcal{R}\left(E_{k}\right)
$$

The energy spectrum $\mathcal{P}\left(E_{k}\right)=\langle\Psi|\hat{\Delta}| \Psi\rangle$, where $\hat{\Delta}$ is the window operator [16]:

$$
\hat{\Delta}\left(E_{k}, n, \epsilon\right) \equiv \frac{\epsilon^{2^{n}}}{\left(\hat{\mathcal{H}}-E_{k}\right)^{2^{n}}+\epsilon^{2^{n}}},
$$

$\hat{\mathcal{H}}$ is the system asymptotic Hamiltonian, and $n$ determines the shape of the window function. As $n$ is increased, this shape rapidly becomes rectangular with very little overlap between adjacent energy bins [16], the bin width remaining constant at $2 \epsilon$. The spectrum is constructed for a set of $E_{k}$ where $E_{k+1}=E_{k}+2 \epsilon$. Thus, scattering information over a range of incident energies can be extracted from a time-dependent wave function that has been calculated on a grid. In this work, $n=2$ and $\epsilon=50 \mathrm{keV}$. Solving two successive linear equations for the vector $\langle\chi\rangle$ :

$$
\left(\hat{\mathcal{H}}-E_{k}+\sqrt{i} \epsilon\right)\left(\hat{\mathcal{H}}-E_{k}-\sqrt{i} \epsilon\right)|\chi\rangle=|\Psi\rangle,
$$

yields $\mathcal{P}\left(E_{k}\right)=\epsilon^{4}\langle\chi \mid \chi\rangle$.

At deep sub-Coulomb energies, $\mathcal{R}\left(E_{k}\right) \approx 1$, and Eq. (4) becomes numerically unstable. The transmission coefficient is then obtained from:

$$
\mathcal{T}\left(E_{k}\right)=\frac{-\left(8 / \hbar v_{k}\right) \epsilon^{4}\langle\chi|\operatorname{Img}(\hat{W})| \chi\rangle}{\mathcal{P}^{\text {initial }}\left(E_{k}\right)},
$$




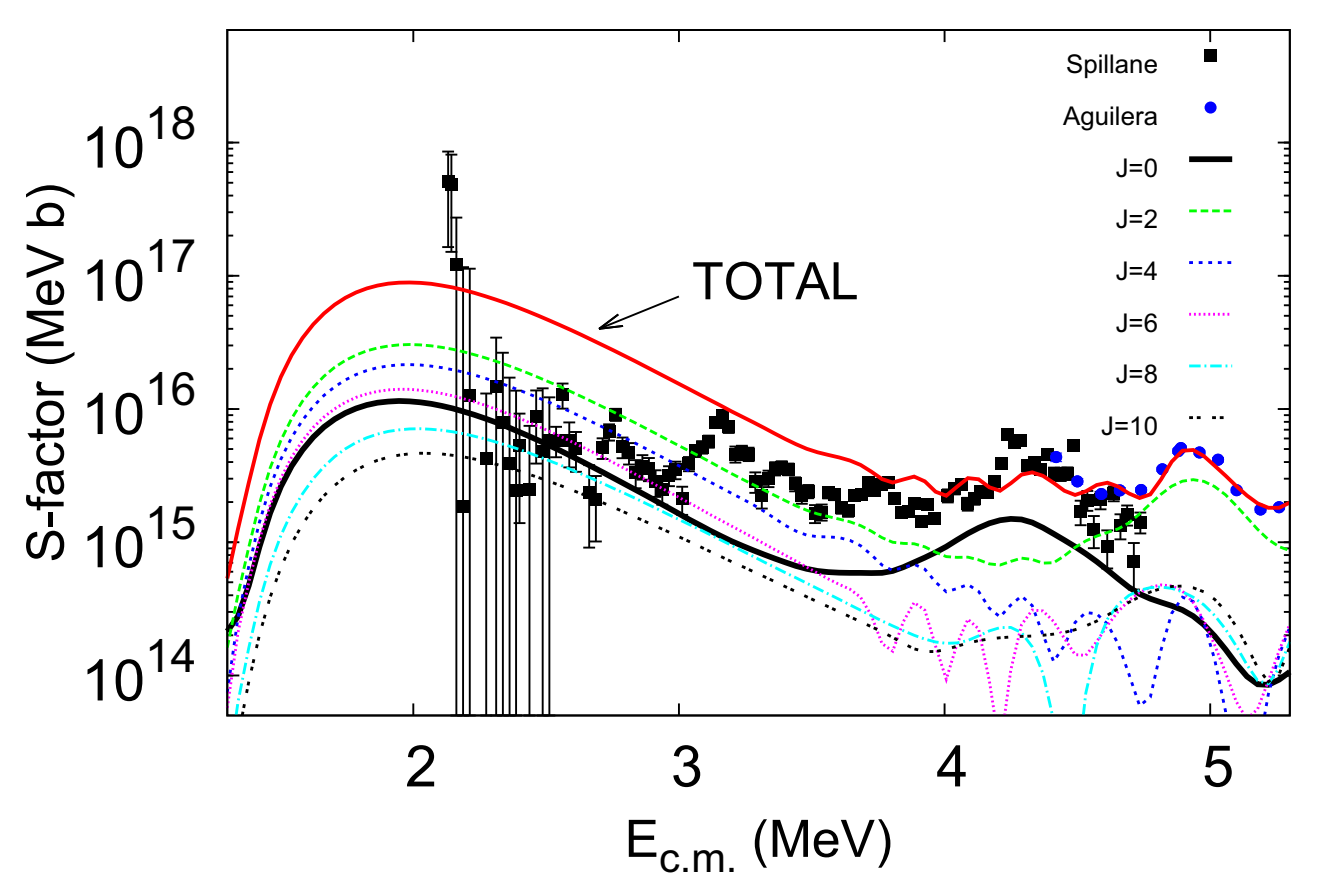

Figure 3. The sub-Coulomb S-factor excitation function for ${ }^{12} \mathrm{C}+{ }^{12} \mathrm{C}$. Measurements $[3,4]$ are compared to preliminary theoretical calculations within the time-dependent wave-packet method, indicating that molecular structure and fusion are closely connected.

where $\operatorname{Img}(\hat{W})<0$ denotes the imaginary potential that operates at the fusion pocket in Fig 1 , while $v_{k}=\sqrt{2 E_{k} / \mu}$ is the asymptotic relative velocity. Eqs. (4) and (7) provide the same results at energies around the Coulomb barrier, which is a good test of the calculation. As an example and sake of simplicity, Fig. 2 shows the transmissioncoefficient excitation function for the ${ }^{16} \mathrm{O}+{ }^{16} \mathrm{O}$ central collision, which is determined by three methods: solving the stationary Schrödinger equation (solid line) and employing Eqs. (4) and (7) (symbols). The good agreement among these methods demonstrates the reliability of the present time-dependent wave-packet approach.

The model calculations are performed on a fivedimensional grid, i.e., a Fourier radial grid $(R=0-1000$ fm) with 2048 evenly spaced points [18], and for the angular variables, $\left(\theta_{1}, k_{1}\right)$ and $\left(\theta_{2}, k_{2}\right)$, a grid based on the extended Legendre discrete-variable representation (KLegDVR) method [19]. The KLeg-DVR grid-size is determined by the values of $j_{\max }$ and $k_{\max }$ [19], which are set as 4 and 0 , respectively. Total angular momenta up to $J=10 \hbar$ are included. The cross sections are provided by a single wave-packet propagation with the initial, average total energy $E_{0}=3 \mathrm{MeV}$. The initial wave-packet was centered at $R_{0}=400 \mathrm{fm}$, with width $\sigma=10 \mathrm{fm}$, and was boosted toward the collective potential-energy landscape in Fig. 1 with the appropriate average kinetic energy for the $E_{0}$ required. The calculations are considered preliminary, as these do not include Coriolis effects yet.

The sub-Coulomb S-factor excitation function for ${ }^{12} \mathrm{C}$ $+{ }^{12} \mathrm{C}$ is presented in Fig. 3. The fusion cross section, $\sigma_{f u s}(E)$, is calculated taken into account the identity of the interacting nuclei and the parity of the radial wave function (only even partial waves $J$ are included), i.e., $\sigma_{\text {fus }}(E)=\pi \hbar^{2} /(2 \mu E) \sum_{J}(2 J+1)\left(1+\delta_{1,2}\right) \mathcal{T}_{J}(E)$, where $\mu$ is the reduced mass, $E$ is the incident energy in the centerof-mass frame and $\mathcal{T}_{J}$ is the partial transmission coefficient. The S-factor is $S(E)=\sigma_{f u s}(E) E \exp (2 \pi \eta)$, where the Sommerfeld parameter $\eta=(\mu / 2)^{1 / 2} Z_{1} Z_{2} e^{2} /\left(\hbar E^{1 / 2}\right)$ and $Z_{i}=6$ is the ${ }^{12} \mathrm{C}$ charge number. Fig. 3 shows important features:

1. The total angular momenta $J=0$ and $2 \hbar$ clearly determine the observed resonant structures in the 4$5 \mathrm{MeV}$ energy window.

2. The $J=0$ component is not dominant at deep subCoulomb energies, where various partial waves substantially contribute to the total S-curve. At these energies, the total S-curve is smooth and overestimates the data. For $E<2 \mathrm{MeV}$, the fusion cross section declines strongly. More complete and precise calculations as well as more accurate measurements are required at the astrophysically important energy region $(E<3 \mathrm{MeV})$, which are challenging and are being pursued currently.

Preliminary calculations are very promising, indicating that molecular structure and fusion are closely connected in both the ${ }^{12} \mathrm{C}+{ }^{12} \mathrm{C}$ and ${ }^{16} \mathrm{O}+{ }^{16} \mathrm{O}$ systems [20]. Calculations for other astrophysically important heavy-ion systems (e.g., ${ }^{16} \mathrm{O}+{ }^{12} \mathrm{C}$ ) are in progress. The present method might be a more suitable tool for expanding the 
cross section predictions towards lower energies than the commonly employed potential-model approach.

\section{References}

[1] C. Rolfs and W.S. Rodney, Cauldrons in the Cosmos (University of Chicago Press, Chicago, 1988).

[2] L. Barron-Palos et al, Nucl. Phys. A 779, 318 (2006

[3] E.F. Aguilera et al, Phys. Rev. C 73, 064601 (2006).

[4] T. Spillane et al, Phys. Rev. Lett. 98, 122501 (2007).

[5] L.R. Gasques et al, Phys. Rev. C 76, 035802 (2007).

[6] R.L. Cooper et al, Ap. J 702, 660 (2009).

[7] M.E. Bennett et al, J. of Phys: Conf. Ser. 202, 012023 (2010).

[8] C.L. Jiang et al, Phys. Rev. Lett. 110, 072701 (2013).

[9] M. Assuncao and P. Descouvemont, Phys. Lett. B 723, 355 (2013).

[10] W. Greiner, J.Y. Park and W. Scheid, Nuclear Molecules (World Scientific Pub, Singapore, 1995).
[11] A. Diaz-Torres and M. Wiescher, J. Phys.: Conf. Ser. 492, 012006 (2014).

[12] K.T.R. Davies and J.R. Nix, Phys. Rev. C 14, 1977 (1976).

[13] A. Diaz-Torres, Phys. Rev. Lett. 101, 122501 (2008).

[14] F. Gatti et al, J. Chem. Phys. 123, 174311 (2005).

[15] V.A. Mandelshtam and H.S. Taylor, J. Chem. Phys. 103, 2903 (1995).

[16] K.J. Schafer and K.C. Kulander, Phys. Rev. A 42, 5794 (1990).

[17] D.J. Tannor, Introduction to Quantum Mechanics: A Time-Dependent Perspective (University Science Books, Saulito, 2007).

[18] R. Kosloff, Ann. Rev. Phys. Chem. 45, 145 (1994).

[19] S. Sukiasyan and H.-D. Meyer, J. Chem. Phys. A 105, 2604 (2001).

[20] A. Diaz-Torres A, L.R. Gasques and M. Wiescher, Phys. Lett. B 652, 25 (2007). 